Banerjee A. K. \& Shatkin. A. J. J. Jinwl 6, I 11 (1970).

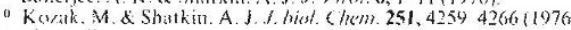

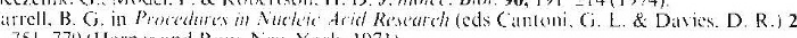

751779 , Harper and Row. Aew York. 1971)

5 Hains, J. A. Reese. C. B. . Todd. A. R. f. chem. Ses . 5281 (1967)

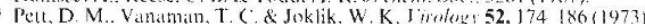

is Legon, S.J.moles. Biol. 1166, 375311976 ).
Rhodes. D. P. \& Banerite. A. K. J.13w. 17,33 +2119761

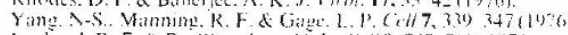

Lockird. R. F. \& RitiBhandary. L 1.. (c/19, 747760 ) 1976

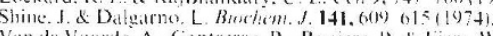

Van de Voorde. A. Contreras. R. Rogiers. R. \& Fier. W. (c/19,117 12011976).

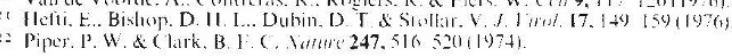

\section{Identification of cosmic $\gamma$-ray sources CG135+1 and CG189+1 with HII regions}

ELEVEN unidentified $\gamma$-ray sources have been reported by the $\operatorname{COS} \mathrm{B}$ group ${ }^{1}$ one of which was previously known from the SAS 2 satellite $^{2}$. Here, I point out two likely associations of the new sources with giant HII regions. CG135+1 lies in the direction of the complex of HII regions, OB associations, molecular clouds and dust in the Cas OB6 region of the Perseus arm. An $\mathrm{H} \alpha$ photograph and identification chart for this region is given in ref. 3. The HII region W3 (IC1795) is located at $\left(I=133.7^{\circ}\right.$, $\left.b=+1.1^{\circ}\right)$, which is only $0.7^{\circ}$ outside the one standard deviation error box for CG135 $1\left(I-135.5^{\circ} \pm 1.0^{\circ}, b=1.5^{\circ} \pm\right.$ $\left.1.0^{\circ}\right)^{1}$. The SAS 2 longitude distribution ${ }^{4}$ is consistent with the existence of this source.

W3 lies at a distance of $\sim 3.1 \mathrm{kpc}$ in the Perseus $\mathrm{arm}^{5}$, and contains 10 infrared sources ${ }^{6.7}$, four condensed centimetre sources W3(A,B,C,D) (ref. 8) and an $\mathrm{OH}$ maser source ${ }^{8}$. A spectrum of W3(A) from $408 \mathrm{MHz}-10^{14} \mathrm{~Hz}$ is given in ref. 9 . Krügel and Mezger ${ }^{10}$ suggest that W3 is an $\mathrm{O}$-star association in a very early evolutionary stage, the component $W 3(A)$ consisting of a compact dense shell of ionised gas surrounded by a dense shell of neutral gas (density $\sim 10^{4} \mathrm{~cm}^{-3}$ ). The total infrared luminosity is $\sim 10^{6} L$ (ref. 11); 1 -mm observations ${ }^{12}$ suggest a column density for W3 of $10^{23} \mathrm{H}_{2}$ molecules $\mathrm{cm}^{-2}$, and an $\mathrm{H}_{2}$ mass of $10^{3} M_{\odot}$.

The reported intensity of CG135 +1 is $\sim 2 \times 10^{-6}$ photons $(>70 \mathrm{MeV}) \mathrm{cm}^{-2} \mathrm{~s}^{-1}$ (ref. 13). If the cosmic-ray proton intensity is the same in W3 as locally, we can estimate the intensity of $\pi^{\prime \prime}$-decay $\gamma$ rays using the source function given by Stecker ${ }^{14}$ to be $\sim 1.6 \times 10^{-10}$ photons $(>70 \mathrm{MeV}) \mathrm{cm}^{-2} \mathrm{~s}^{-1}$. Bremsstrahlung interactions may increase this by up to a factor $\sim 2$ (ref. 15) if the $\mathrm{e} / \mathrm{p}$ ratio is the same in the cosmic-radiation flux near W3 as locally. Thus if the identification is correct, a density of cosmic radiation $\sim 10^{4} \times$ the local density is required; this might be plausible if the sources of cosmic rays were associated with the young stars in the HII regions. The particles could either have been accelerated in the stars themselves, or in an associated supernova or SNR (the SNR HB3 is nearby and may be physically connected with $W 3^{16,17}$ ). The association of $W 3$ with the $\gamma$-ray source, however, could be attributed to an unseen $\gamma$-ray pulsar (there is no known radio pulsar in this direction) or an unknown $\gamma$-ray emitter connected with young stellar populations.

I also suggest the identification of the source CG189+1 $\left(l-189.0^{\prime \prime} \pm 1.5^{\circ}, \quad b=1.0^{\circ} \perp 1.5^{\circ}\right)^{\prime}$ with the HII region NGC2175 at $/=190^{\circ}, b=0.5^{\circ}$. Aperture synthesis observations of this object, and a report of an associated $\mathrm{CO}$ cloud $20 \mathrm{pc}$ in diameter, have been published ${ }^{18}$.

NGC2175 has the same $6-\mathrm{cm}$ flux density $(30 \mathrm{Jy})$ as W3(A $)^{19}$ and should have a similar infrared intensity to W3(A) if the proportionality of infrared to centimetre continuum applies". The positional coincidence here is in fact better than for W3.
The $\gamma$-ray source is extended in latitude ${ }^{1}$, and also includes the direction to the SNR IC $443\left(l=189^{\circ}, b-3.2^{2}\right)^{2 * t}$ which is an X-ray source ${ }^{23}$. The distance to both IC 443 and NGC 1275 is about $1.5 \mathrm{kpc}$, so that the possibility of a physical connection is not ruled out, in which case the situation may provide a case similar to that for W3 and HB3 discussed above.

If $\gamma$-ray emission is a common feature of objects like W3, other objects with similar radio and infrared fluxes should be $\gamma$-ray sources. Of the objects listed in refs 7 and 11 , only W75 lies within the four areas examined by the COS B group ${ }^{1}$. W75 $\left(l-81.9^{\circ}, b-0.8^{\circ}\right)$ lies in the very densely populated Cygnus region in which the visibility of individual sources is rather low. The nearest $\gamma$-ray source is CG78.1 $\left(l=78.5^{\circ}+\right.$ $\left.0.5^{\circ}, b=1.5^{\circ} \pm 0.5^{\circ}\right)^{1}$.

The Orion complex (M42) should be easily observable if the W3 identification is correct, since it is about five times as intense both at $30-300 \mu \mathrm{m}$ and at $2 \mathrm{~cm}$ (ref. 11), and is in a region away from the Galactic plane where point-source visibility should be good. But it lies outside the four regions examined by Hermsen et al. ${ }^{1}$, M17 is also a good candidate, being brighter than W3 in both infrared and radio; however, it is in the galactic plane at longitude $14.7^{\circ}$, and hence harder to distinguish from the galactic background.

I thank Professor A. W. Wolfendale for useful discussions and the Royal Commission for the Exhibition of 1851 for the provision of a Fellowship.

\section{A. W. STRONG}

Physics Department,

University of Durham,

Durham, UK

Received 20 June; accepted 15 August 1977.

1. Hermsen, W. et al. 12th ESLAB Symp. (ed. Battrick, B.) (ESTEC, Noordwijk,

1977).
Thompson, D. J., Fichtel, C. E., Hartman, R. C., Kniffen, D. A. \& Lamb. R. C. Astrophys. J. 213, 252-262(1977).

. Georgelin, Y. M. \& Georgelin, Y.P. Astr. Astrophys. 49, 57-79 (1976)

4. Fichtel, C. E. Kniffen, D. A. \& Thompson, D. J. I2th ESI.AB Symp. (ed. Battrick, B.) (ESTFC, Noordwijk, 1977).

Reifenstein III, E. C. Wilson, T. L., Burke, B. F., Mezger, P. G. \& Altenhoff, W. J. Astr. Astrophys, 4, 357-377 (i970)

6. Wynn-Williams, C. G., Becklin, E. E. \& Neugebauer, G. Mon. Not. R. ustr. Soc $160,1-14$ (1972).

. Dyck, H. M. \& Simon, T. Astrophys. J. 211, 421-428 (1977)

8. Wynn-Williams, C. G. Mon. Not. R. ustr. Soc 151, 397 (1971),

. Mezger, P. G. in Fur Infra-red Astronomy (ed. Rowan-Robinson, M.)(Pergamon.

10. Krügel, E. \& Mezger, P. G. Astr. Astroptivs. 42, 441-448 (1975).

11. Harper D. Astrophys J 192,557.571 (1974).

2. Westbrook, W. E. et al. Astrophys, J. 209,94-101 (1976).

Wills, R. D. IAU Circ No. $2992(1976)$.

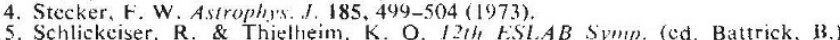
(ESTFC Noordwijk 1977).

(ESTFC, Noordwijk. 1977)

16. C.aswell, J. L. Mon. Not. R. ustr. Soc, 136, 11-18 (1967)

17. Caswell. J. L. Mon. Not. R astr. Soc 177,601-616(1976)

18. Felli, M. Ilabing, H. J. \& Isracl, F. P. Astr. Astrophys, 59, 43-52 (1977).

19. Gevel. W. L. Astrophys, $1.153,743-760(1968)$

21. Winkler, P. F. \& Clark, G. W. Astrophys. J. Lett. L67-L69 (1974) 